\title{
Antibiotikaprophylaxe in der Orthopädie und Unfallchirurgie - was, wann und wie lange applizieren?
}

\author{
Christian Kleber, Andrej Trampuz
}

\section{Zusammenfassung}

Durch korrekte perioperative Antibiotikaprophylaxe kann die Inzidenz von postoperativen Infektionen deutlich gesenkt werden. Eine einmalige Antibiotikadosis genügt bei Operationen bis zu einer Dauer von 3 Stunden, eine 2. Dosis soll gegeben werden, wenn der Eingriff $>3$ Stunden dauert. Eine Verlängerung der Antibiotikaprophylaxe auf $>24$ Stunden erbringt keinen zusätzlichen Nutzen, birgt aber die Gefahr einer Resistenzentwicklung und häuft die Nebenwirkungen. Cephalosporine der 1 . ( $2 \mathrm{~g}$ Cefazolin) oder 2. Generation (1,5 g Cefuroxim) sollten 30-60 Minuten vor Schnitt verabreicht werden. Bei Penicillinallergie vom Typ I (Anaphylaxie), bei Cephalosporinallergie oder einer hohen Rate vom Methicillin-resistenten Staphylococcus aureus (MRSA) soll Vancomycin $(1 \mathrm{~g})$ eingesetzt werden.
Antibiotic Prophylaxis and Therapy in Orthopaedic and Traumatology Surgery - What, When and How Long to Administer?

The incidence of postoperative infections can be significantly reduced with correct perioperative antibiotic prophylaxis. A single dose of an antibiotic is sufficient for surgeries of less than 3 hours, a second dose should be given in surgeries lasting $>3$ hours. Antibiotic prophylaxis of $>24$ hours has no additional effect, however, the resistance pressure and adverse effects are increased. Cephalosporins of the first ( $2 \mathrm{~g}$ cefazolin) or second generation ( $1.5 \mathrm{~g}$ cefuroxim) should be given 30 $60 \mathrm{~min}$ prior to the first incision. In cases of a penicillin allergy of type I (anaphylaxyis), cephalosporin allergy or a high rate of methicillin-resistant Staphylococcus aureus (MRSA) vancomycin (1 g) should be used.

\section{Grundlagen der perioperativen Antibiotikaprophylaxe}

Infektionen im Operationsgebiet gehören zu den bedeutendsten Komplikationen nach chirurgischen Eingriffen. Sie führen oft zu verlängerten Krankenhausaufenthalten, erhöhter Morbidität und hohen Behandlungskosten [1]. Die meisten Infektionen entstehen während der Operation durch direkte Besiedlung (Kolonisation) des Fremdmaterials. Hierfür spricht die Effizienz präventiver Maßnahmen, wie der perioperativen Antibiotikaprophylaxe und konsequente Hautdesinfektion, durch welche die Rate postoperativer Infektionen deutlich reduziert werden konnte. Die Antibiotika-

OP-JOURNAL 2014; 30: 8-10

(C) Georg Thieme Verlag KG Stuttgart · New York DOI http://dx.doi.org/10.1055/s-0034-1368332 prophylaxe hat sich deshalb für definierte orthopädische und unfallchirurgische Eingriffe breit durchgesetzt und wurde in nationale und internationale Leitlinien umgesetzt [2].

Die perioperativen Infektionen werden in erster Linie durch Bakterien der Hautflora des Patienten und der Raumluft verursacht. Eine Vielzahl dieser Infektionen ist vermeidbar, wobei zu den wichtigsten Maßnahmen zur Reduktion perioperativer Infektionen die korrekte präoperative Antibiotikaprophylaxe gehört [3]. Eine effiziente und optimal eingesetzte Antibiotikaprophylaxe ist jedoch kein Ersatz für eine gute Operationstechnik oder ein kompetentes prä- und postoperatives Management. Ist der Patient z. B. an einer systemischen oder lokalen Infektion erkrankt, so sollte diese zuerst erkannt und behandelt und eine elektive Operation verschoben werden. Bei Dia- betikern sollte die Blutzuckereinstellung optimal sein und der Patient sollte gut oxygeniert und nicht hypotherm sein. Das präoperative Duschen mit einem Antiseptikum (z.B. Chlorhexidin oder Octenidin) reduziert die Keimzahl auf der Haut und die Häufigkeit von postoperativen Infektionen.

\section{Definitionen zum Antibiotikaeinsatz}

- Prophylaxe: Antibiotikum gelangt vor dem Erreger in die Wunde. Beispiel: perioperative Antibiotikaprophylaxe, in der Regel Einzeldosis 30-60 Minuten vor Schnitt.

- Präemptive Therapie: Antibiotikum gelangt erst nach dem Erreger, jedoch vor der Infektion in die operative Wunde. Beispiel: Antibiotikagabe nach offener Fraktur, in der Regel für eine Dauer von 5 Tagen.

- Empirische (kalkulierte) Therapie: Antibiotikagabe ohne Kenntnis der Erreger, aufgrund von Erfahrungswerten und lokaler Epidemiologie. Beispiel: Therapie nach Explantation einer infizierten Gelenkprothese.

- Gezielte Therapie: Antibiotikagabe aufgrund eines definierten Keimes mit nachgewiesener Empfindlichkeit (Antibiogramm).

\section{Das Implantat und der Biofilm}

Wenn ein Implantat operativ eingebracht wird, sind schon wenige Hunderte an Bakterien ausreichend, eine Biofilmbildung und Infektion auszulösen. Der Biofilm ist eine Strategie der Bakterien und Pilze, auf Fremdkörpern zu überleben und die eigene Kolonie zu erhalten. Der Biofilm ist das Resultat der Evolution und der Anpassung von Mikroorganismen an ihr Habitat. Mehr als $80 \%$ aller Mikroorganismen in der Natur existieren als Biofilm. Ein reifer Biofilm setzt sich zu 25-30\% aus Bakterien und 70-75\% aus amorpher Matrix zusammen (Abb. 1). 


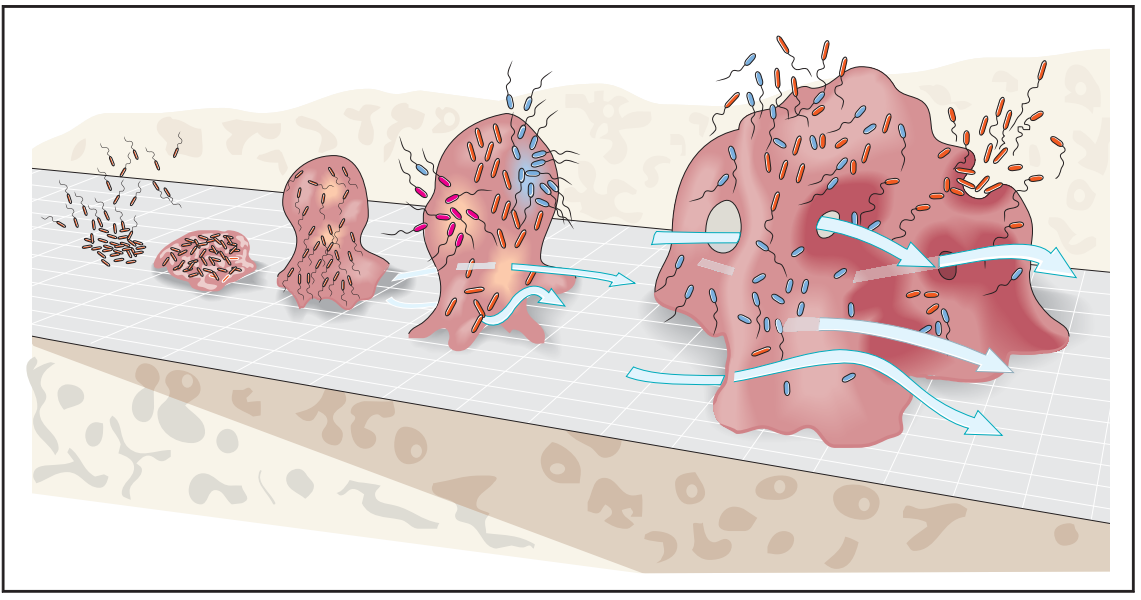

Abb. 1 Biofilmbildung auf der Implantatoberfläche. Planktonische Bakterien adhärieren auf die Oberfläche und bilden eine amorphe Matrix, in welcher sie als Biofilm eingebettet sind. Der Biofilm hat nach wenigen Tagen eine 3-dimensionale Struktur.

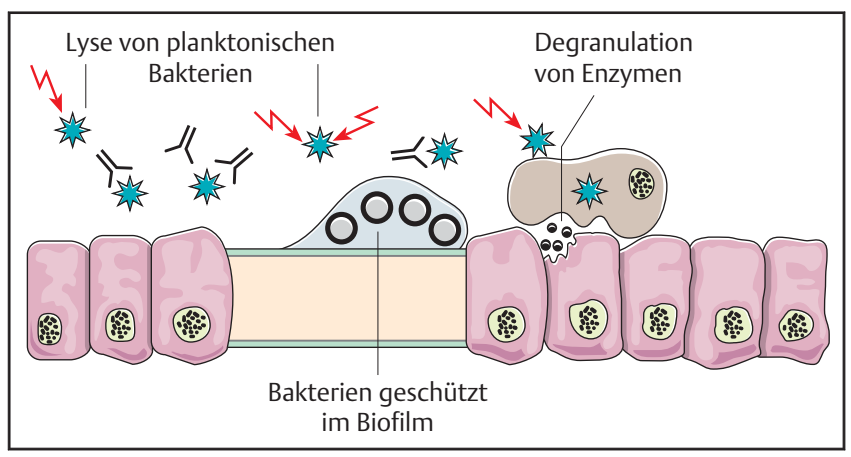

Abb. 2 Frei lebende (planktonische) Bakterien werden von Antibiotika und dem Immunsystem abgetötet, während adhärente Bakterien in der extrazellulären Matrix des Biofilms geschützt überleben.

Mit einer Antibiotikaprophylaxe wird der erste Schritt der Biofilmbildung auf Implantaten verhindert: die Adhäsion.

Nach der Adhäsion vermehren sich Mikroorganismen auf der Oberfläche und bilden in wenigen Stunden eine mehrschichtige Struktur (früher Biofilm). Im weiteren Verlauf entwickelt sich eine stabile Zellmatrix (reifer Biofilm), gefolgt von der Ablösung einzelner Mikroorganismen von der Oberfläche. In der Biofilmmatrix befinden sich die Bakterien in reduzierter metabolischer Aktivität (stationäre Wachstumsphase), weswegen sie bis zu 1000-mal resistenter gegen die meisten Antibiotika sind (Abb. 2) [4,5]. Die Ausnahme stellen das Rifampicin (bei Staphylokokken) und die Chinolone (bei gramnegativen Stäbchen) dar, welche eine intrinsische Wirkung gegen Biofilme besitzen. Deswegen werden diese Antibiotika bei einer Eradikationsbehandlung von implantatassoziierten Infektionen eingesetzt und sollten nicht in der Antibiotikaprophylaxe angewendet werden [2].
Weil der erste Schritt der implantatassoziierten Infektionen die Adhäsion von Mikroorganismen an die FremdkörperOberfläche erfordert, kann durch eine antimikrobielle Beschichtung (z.B. Silber, Chlorhexidin, Antibiotika) oder Veränderung der Implantatoberfläche (z.B. Nanostruktur) die Haftung der Bakterien vermindert und somit das Risiko einer Infektion reduziert werden. Auch die Antibiotikaprophylaxe wirkt durch Verauf der Implantatoberfläche. Deswegen muss die Prophylaxe in hoher Dosis vor dem chirurgischen Eingriff verabreicht werden, sodass zum Zeitpunkt der Implantation eine ausreichend hohe Konzentration am Operationsort vorhanden ist, bevor potenzielle Bakterien in Kontakt mit dem Implantat treten [3].

\section{Praktische Empfehlungen zur perioperativen Antibiotikaprophylaxe}

\section{Anzahl von Antibiotikagaben:}

Eine einmalige Antibiotikadosis (sog. „single-shot“) genügt bei Operationen bis zu einer Dauer von 3 Stunden. hinderung der Adhäsion von Bakterien
Einige Zentren verlängern die Prophylaxe auf 24 Stunden. Eine Verlängerung der Antibiotikaprophylaxe auf $>24$ Stunden erbringt keinen zusätzlichen Nutzen, birgt aber die Gefahr einer Resistenzentwicklung und Häufung der Nebenwirkungen. Bei Operationen $>3 \mathrm{~h}$ soll eine zusätzliche Prophylaxegabe verabreicht werden, um zu gewährleisten, dass beim Wundverschluss der Gewebespiegel des Antibiotikums weiterhin genügend hoch ist.

2. Wissenschaftliche Evidenz: Die Effizienz der Antibiotikaprophylaxe in der orthopädischen Chirurgie ist gut belegt. In einer randomisierten Studie mit 2137 Patienten, die eine Hüftprothese erhielten, betrug die Infektionsrate unter Placebo 3,3\% (35/ 1067) und unter Cefazolin 0,9\% (10/ 1070) [6]. Weitere Studien und Metaanalysen haben die Wirksamkeit einer Antibiotikaprophylaxe bei orthopädischen und unfallchirurgischen Eingriffen eindeutig bestätigt und wird daher in allen nationalen und internationalen Leitlinien empfohlen.

3. Zeitpunkt der Antibiotikagabe: Die Antibiotikaprophylaxe soll sicherstellen, dass vom Zeitpunkt der Hautinzision bis zum Wundverschluss bakterizide Konzentrationen im Gewebe des OP-Situs bestehen [1]. In einer prospektiven Beobachtungsstudie mit 3836 chirurgischen Eingriffen und einer Prophylaxe mit 1,5 g Cefuroxim i.v. wurde bestätigt, dass mit einer Prophylaxegabe 30-59 Minuten vor Inzision signifikant tiefere Wundinfektionsraten zu verzeichnen waren als bei einer Gabe während der letzten 30 Minuten [7]. In einer prospektiven Studie mit 2847 Patienten wurden die Wundinfektionsraten in Relation zum Zeitpunkt der Gabe prophylaktischer Antibiotika evaluiert. Während die Wundinfektionsrate bei Gabe der Prophylaxe innerhalb von 2 Stunden vor Schnitt mit 0,6\% tief war, lagen die Raten bei früherer Gabe mit 2,4\% oder späterer Gabe mit 3,3\% deutlich höher [8]. Aufgrund tierexperimenteller und klinischer Studien ist die Infektionsrate am niedrigsten, wenn der Zeitpunkt der Antibiotikagabe mit der Narkoseeinleitung zusammenfällt (30-59 Minuten vor Inzision) [7]. Bei Operationen in Blutsperre (z. B. Kniearthroplastik) wurde bisher die Antibiotikaprophylaxe 30-60 Minuten vor Insufflation des Tourniquets verabreicht. In einer Studie an 24 Patienten mit einer Kniearthroplastik wurde $1 \mathrm{~g}$ Cefazolin 1, 2 
Tab. 1 Empfohlene perioperative Prophylaxe nach Art des Eingriffs und Schweregrad des Weichteilschadens nach Gustilo/Anderson bei offenen Frakturen.

\begin{tabular}{|c|c|}
\hline Indikation & Antibiotikum und Verabreichung* \\
\hline $\begin{array}{l}\text { alle Operationen mit Osteosynthesen- } \\
\text { oder Prothesen bei Narkoseeinleitung } \\
\text { (mit Ausnahme reiner perkutaner Kirsch- } \\
\text { ner-Draht-Osteosynthesen oder Osteo- } \\
\text { synthesen ausschließlich mit Schrauben) }\end{array}$ & $\begin{array}{l}2 \mathrm{~g} \text { Cefazolin oder } 1,5 \mathrm{~g} \text { Cefuroxim i. v. vor Ope- } \\
\text { ration (in der Regel als Einzeldosis) } \\
\text { falls Blutsperre: } 30-60 \text { Minuten vor Schließen } \\
\text { der Blutsperre }\end{array}$ \\
\hline offene Frakturen 1. und 2. Grades & $\begin{array}{l}2 \mathrm{~g} \text { Cefazolin oder } 1,5 \mathrm{~g} \text { Cefuroxim i. v. vor Ope- } \\
\text { ration (Einzeldosis) oder während } 24 \text { Stunden }\end{array}$ \\
\hline offene Frakturen 3. Grades & $\begin{array}{l}\text { Ampicillin-Sulbactam } 3 \mathrm{~g} \text { alle } 8 \text { Stunden i.v. oder } \\
\text { Piperacillin-Tazobactam } 4,5 \mathrm{~g} \text { alle } 8 \text { Stunden } \\
\text { i. v. für } 5 \text { Tage }\end{array}$ \\
\hline $\begin{array}{l}\text { nach Antibiotikatherapie von über } \\
2 \text { Wochen vor der Operation }\end{array}$ & $\begin{array}{l}\text { zusätzlich zur üblichen Prophylaxe: Vancomycin } \\
1 \mathrm{~g} \text { i.v. als Einzeldosis Beginn } 2 \text { Stunden vor } \\
\text { Schnitt als langsame Infusion (über } 1 \text { Stunde) }\end{array}$ \\
\hline $\begin{array}{l}\text { Ausbau des Implantats (ohne einzeitigem } \\
\text { Implantat-Wiedereinbau) }\end{array}$ & $\begin{array}{l}\text { keine Prophylaxe notwendig } \\
\text { bei Verdacht auf Infektion: unmittelbar nach } \\
\text { Probenentnahme Beginn mit empirischer Anti- } \\
\text { biotikatherapie }\end{array}$ \\
\hline
\end{tabular}

* Gabe als Kurzinfusion 30-60 Minuten vor Inzision. Bei Eingriffen von über 3 Stunden Dauer soll eine weitere Dosis des jeweiligen Antibiotikums intraoperativ verabreicht werden.

oder 5 Minuten vor Tourniquetinsufflation verabreicht. Genügend hohe Gewebe- und Knochenkonzentrationen fanden sich lediglich bei Patienten mit einer Gabe 5 Minuten vor Anlegen der Blutsperre [9].

\section{Antibiotikaauswahl:}

Cephalosporine der 1. (Cefazolin) oder 2. Generation (Cefuroxim) haben sich bei Eingriffen am Knochen gut etabliert.

Das Spektrum der Cephalosporine ist gegen die häufigsten Erreger (Staphylokokken, Streptokokken und grampositive Anaerobier wie Propionibacterium acnes) wirksam. Bei Allergie vom Nichttyp I können weiterhin Cephalosporine appliziert werden. Bei Penicillinallergie vom Typ I (Anaphylaxie), bei Cephalosporinallergie oder einer hohen Rate vom Methicillin-resistenten Staphylococcus aureus (MRSA) soll Vancomycin eingesetzt werden, welches jedoch den Cephalosporinen bez. Wirkung und Nebenwirkungen unterlegen ist. Antibiotika mit einem breiten Spektrum (Imipenem, Meropenem, Ertapenem, PiperacillinTazobactam) sind den Cephalosporinen bei der perioperativen Prophylaxe nicht überlegen. Sie werden als Therapiereserven bei Infektionen eingesetzt und sollen deshalb nicht zur Prophylaxe verwendet werden.

\section{Praktisches Vorgehen}

Unter einer länger dauernden Antibiotikatherapie kann es zur Selektion von Methicillin-resistenten Koagulase-negativen Staphylokokken kommen. Bei Patienten mit vorangegangener Antibiotikatherapie von über 2 Wochen oder bekanntem MRSA-Trägertum soll vor Implantation von Osteosynthesematerial oder Endoprothese zusätzlich zur regulären Prophylaxe Vancomycin 1 g 2 Stunden (single dose) erwogen werden (Infusionsdauer 60 Minuten).

Bei folgenden Eingriffen gibt es keine Evidenz für die Wirkung einer Antibiotikaprophylaxe; diese sollte deswegen vermieden werden:

- arthroskopische Eingriffe ohne Einbringen von Fremdmaterial

- Eingriffe an der Wirbelsäule ohne Einbringen oder Vorhandensein von Implantaten

- Arthrotomie
- Amputationen, wenn nicht durch Infektion bedingt

- Entfernung von Osteosynthesematerial

In Tab. 1 finden Sie eine Zusammenfassung des Artikels und aktuelle Leitlinie der perioperativen Antibiotikaprophylaxe unseres Centrums für Muskuloskeletale Chirurgie.

\section{Literatur}

1 Trampuz A, Widmer AF. Infections associated with orthopaedic implants. Curr Opin Infect Dis 2006; 19: 349-356

2 Zimmerli W, Trampuz A, Ochsner PE. Prosthetic-joint infections. N Engl J Med 2004; 351: 1645-1654

${ }^{3}$ Marculescu CE, Osmon DR. Antibiotic prophylaxis in orthopaedic surgery. Infect Dis Clin North Am 2005; 19: 931-936

4 Trampuz A, Perka C, Borens O. [Prosthetic joint infection: new developments in diagnosis and treatment]. Dtsch Med Wochenschr 2013; 138: 1571-1573

5 Winkler T, Trampuz A, Hardt S et al. [Periprosthetic infection after hip arthroplasty]. Orthopade 2014; 43: 70-78

${ }^{6}$ Hill C, Flamant R, Mazas F et al. Prophylactic cefazolin versus placebo in total hip replacement. Report of a multicenter doubleblind randomized trial. Lancet 1981; 8224: 795796

7 Weber WP, Marti WR, Zwahlen M et al. The timing of surgical antimicrobial prophylaxis. Ann Surg 2008; 247: 918-926

8 Classen DC, Evans RS, Pestotnik SL et al. The timing of prophylactic administration of antibiotics and the risk of surgical-wound infection. N Engl J Med 1992; 326: 281-286

${ }^{9}$ Friedman RJ, Friedrich $L V$, White RL et al. Antibiotic prophylaxis and tourniquet inflation in total knee arthroplasty. Clin Orthop Relat Res 1990; 260: 17-23

\section{Dr. med. Christian Kleber}

Facharzt für Orthopädie und

Unfallchirurgie

Operative Intensiv- und

Notfallmedizin

Priv.-Doz. Dr. med. Andrej Trampuz

Facharzt für Infektionskrankheiten

Charité - Universitätsmedizin Berlin

Zentrum für septische Chirurgie

Centrum für Muskuloskeletale

Chirurgie

Campus Virchow Klinikum

Augustenburger Platz 1

13353 Berlin

andrej.trampuz@charite.de 